\title{
Regionalentwicklung, Migration und Fläche
}

\author{
Philipp Eichenauer · Andreas Klee $\cdot$ Hendrikje Wehnert
}

(C) Springer-Verlag Berlin Heidelberg 2013

Städtische und ländliche Räume können bei wirtschaftlicher Prosperität Menschen anziehen. Umgekehrt können Regionen bei ungünstigen Bedingungen Bevölkerung verlieren. Sicherlich muss diesen Aussagen insofern zugestimmt werden, als die Attraktivität einer Region von einer Vielzahl an unterschiedlichen Faktoren bestimmt wird und nicht auf einen Aspekt reduziert werden kann. Nichtsdestotrotz soll dadurch angedeutet werden, dass ein Faktor einen verstärkenden Einfluss auf die Entwicklung einer Region hat: die Migration von Menschen. Neben dem Verhältnis von Geburten- und Sterberate, der natürlichen Bevölkerungsentwicklung, bestimmt sie die demographische Entwicklung eines Raumes. Migration kann sowohl positive wie negative Folgen für die räumliche Entwicklung haben. Im Rahmen dieser Ausgabe der Zeitschrift „Raumforschung und Raumordnung" liegt ein Fokus eher auf dem Phänomen der Abwanderung und ihrer Bedeutung für die Regionalentwicklung. Die Migration spezifischer Altersgruppen kann sich besonders negativ auswirken. In ländlichen Regionen sind daneben geschlechtsspezifische Unterschiede in den Wanderungsmustern festzustellen. Das Fehlen junger Frauen stellt ein nicht unerhebliches Problem für die zukünf-

\footnotetext{
Dr. A. Klee $(\bowtie) \cdot$ P. Eichenauer

Akademie für Raumforschung und Landesplanung -

Leibniz-Forum für Raumwissenschaften, Hohenzollernstraße 11,

30161 Hannover, Deutschland

E-Mail: klee@arl-net.de

P. Eichenauer

E-Mail: eichenauer@arl-net.de

H. Wehnert

Leibniz-Institut für ökologische Raumentwicklung,

Weberplatz 1, 01217 Dresden, Deutschland

E-Mail: h.wehnert@ioer.de
}

tige Regionalentwicklung in Teilen Ostdeutschlands dar. Abwanderung oder gar die Herausbildung einer „Abwanderungskultur" kann ferner die zukünftige demographische, ökonomische und soziale Entwicklung von Räumen entscheidend beeinflussen.

In diese Richtung geht der Beitrag von Karin Wiest und Tim Leibert. Sie untersuchen geschlechtsspezifische Wanderungsmuster im ländlichen Raum. Am Beispiel Sachsen-Anhalt zeigen sie auf, dass die arbeitsmarktinduzierte Abwanderung junger Frauen zu einer unausgewogenen Geschlechterproportion führt. Der Aufsatz erörtert die spezifischen Wanderungsmotive und schlägt Strategien für individuelle Zielgruppen sowie unterschiedliche Politikfelder vor, die einer „Abwanderungskultur“ entgegenwirken sollen. Eine stärkere Identifikation junger Menschen mit ihrer Heimat beispielsweise kann eine bedeutende Bindungswirkung entfalten. Weiter spielen soziale und technische Infrastruktur im ländlichen Raum eine große Rolle. Die Autoren kommen zu dem Ergebnis, dass unausgewogene Geschlechterproportionen ein Leitziel der Raumordnung gefährden, nämlich das der gleichwertigen Lebensverhältnisse.

Dem Phänomen des Wohnungsneubaus in Schrumpfungsregionen widmet sich der Beitrag von Irene Iwanow und Susann Schäfer. Bei sinkenden Einwohnerzahlen findet Wohnungsneu- und Wohnungsrückbau parallel statt, wie exemplarisch an der Entwicklung im Freistaat Sachsen gezeigt wird. Aufgrund der veränderten wirtschaftlichen und sozialen Rahmenbedingungen kommt es in jüngerer Vergangenheit $\mathrm{zu}$ einem Absinken der durchschnittlichen Haushaltsgröße. Dahinter steht der Trend einer Singularisierung der Haushalte, was trotz abnehmender Bevölkerung zu einer Zunahme der Haushalte führt. Wohnungsneubau wird es auch zukünftig in Schrumpfungsregionen geben - wenn auch intraregional sehr unterschiedlich -, um die Wohn- 
wünsche der Einwohner zu befriedigen. Dies muss verstärkt vor dem Hintergrund der demographischen Veränderungen sowie altersspezifischer Wanderungsmuster betrachtet werden. Die Grundlage dafür können kleinräumige Analysen und Wohnungsmarktprognosen liefern, die die kommunale Nachfrage- und Angebotsentwicklung im Blick haben.

Gotthard Meinel erörtert Handlungsmöglichkeiten ,auf dem Weg zu einer besseren Flächennutzungsstatistik“. Die derzeitige amtliche Flächenstatistik offenbart Probleme angesichts der stetig wachsenden Anforderungen bezüglich Genauigkeit, Verlässlichkeit, Aktualität und Aussagekraft. Problematisch sind etwa Veränderungen der Datengrundlagen, die ein Flächennutzungsmonitoring erschweren. Dagegen bietet die Nutzung topographischer Geobasisdaten Vorteile für die Flächenerhebung: Sie sind aktueller, fehlerfreier und finden heute bereits eine breite Anwendung. Dem stellt der Autor im Kontext des Flächennutzungsmonitorings das amtliche Kataster der Geotopographie gegenüber und unterstreicht die Vorteile der topographischen Datengrundlage hinsichtlich räumlicher und zeitlicher Ausdifferenzierung. So versprechen flächendeckende Aktualisierung, breite Anwendung und öffentlicher Zugang eine vergleichsweise hohe Qualität der Flächennutzungsinformation. Abschließend spricht der Autor Empfehlungen für eine zukünftige Flächenstatistik aus.
Das Autorenquintett Jana Bovet, Kilian Bizer, Ralph Henger, Katrin Ostertag und Stefan Siedentop beleuchtet das Konzept der handelbaren Flächenzertifikate. Die Reduktion des Verbrauchs an Siedlungs- und Verkehrsfläche auf 30 Hektar pro Tag ist zwar seit nunmehr zehn Jahren politisch beschlossen, allein die instrumentelle Umsetzung in der Raumplanungspraxis ist umstritten. Abhilfe könnten handelbare Flächenzertifikate leisten, die seit über 15 Jahren in Wissenschaft und Politik diskutiert werden. Bisher wurden zwei Fallstudien mit dem Untersuchungsziel durchgeführt, den Zertifikatehandel in den Verwaltungsablauf $\mathrm{zu}$ integrieren. In enger Kooperation mit den Kommunen wurden fiskalische Wirksamkeitsanalysen von Baulandstrategien erarbeitet, die konkrete Hinweise zu Effizienzgewinnen durch das Instrument liefern. Die Erfahrungen aus den beiden Planspielen fließen in einen bundesweiten Modellversuch ein. Dieses Ende 2012 begonnene Forschungsvorhaben soll weitere Erkenntnisse zur Praxistauglichkeit handelbarer Flächenzertifikate als Instrument liefern.

Wir hoffen, unseren Leserinnen und Lesern wieder eine bereichernde Zusammenstellung von wissenschaftlichen Beiträgen und Berichten anzubieten und wünschen eine kurzweilige Lektüre. 